VOL. 60 (1999) [221-226]

\title{
FINITELY PRESENTED CENTRE-BY-METABELIAN LIE ALGEBRAS
}

\author{
R.M. Bryant and J.R.J. Groves
}

\section{To Bernhard Neumann on his ninetieth birthday}

It is shown that finitely presented centre-by-metabelian Lie algebras are Abelianby-finite-dimensional.

\section{INTRODUCTION}

In [7], the second author proved that a finitely presented centre-by-metabelian group is Abelian-by-polycyclic. The proof of this result used the fact, proved by Bieri and Strebel in [2], that a finitely presented soluble group with an infinite cyclic quotient is an HNN extension with finitely generated base group. In [3], Bieri and Strebel deduced another proof of the result of [7] as a corollary of their work on finitely presented soluble groups, particularly the fact that a metabelian quotient of a finitely presented solubie group is again finitely presented.

The aim of this note is to prove a similar result for Lie algebras.

ThEOREM. A finitely presented centre-by-metabelian Lie algebra is Abelian-byfinite-dimensional.

The key tools quoted above do not seem to be available for Lie algebras. The closest result of which we are aware is one of Wasserman [8, Theorem 9.1] which is similar to the result quoted from [2]. But the consequences of this result do not appear to be sufficiently powerful to obtain results for Lie algebras analogous to those for groups. We have therefore needed to take a substantially different approach.

The authors have shown in [6] that a finitely presented soluble Lie algebra of characteristic 2 which satisfies the maximal condition for ideals must be of finite dimension. Because a finitely generated Abelian-by-finite-dimensional Lie algebra must satisfy the maximal condition for ideals [1, Corollary 11.1.8], the Theorem implies that all finitely presented centre-by-metabelian Lie algebras of characteristic 2 are of finite dimension.

The main step in the proof of the Theorem is to show that a metabelian quotient of a finitely presented centre-by-metabelian Lie algebra is again finitely presented. It would be interesting to know to what extent this can be generalised.

Received 29th April, 1999

Work supported by ARC research grant $\mathrm{S} 69813080$ and by a Royal Society travel grant.

Copyright Clearance Centre, Inc. Serial-fee code: 0004-9727/99 \$A2.00+0.00. 
QUESTION. Is it true that a metabelian quotient of a finitely presented soluble Lie algebra is again finitely presented?

An affirmative answer to the corresponding question for groups is given by [3, Theorem B].

\section{QUOTIENTS OF FINITELY PRESENTED LIE ALGEBRAS}

Throughout this paper $K$ denotes an arbitrary field, and all tensor and exterior products are taken over $K$. If $L$ is any Lie algebra over $K$ we write $K[L]$ for the enveloping algebra of $L$. Also, we write $L^{\prime}$ for the subalgebra $[L, L]$ and $L^{\prime \prime}$ for $\left[L^{\prime}, L^{\prime}\right]$.

Let $L$ be a finitely presented Lie algebra over $K$, and suppose that $A$ and $B$ are ideals of $L$ such that $B \subseteq A$ and $A / B$ is Abelian. Set $R=K[L / A]$ and $M=A / B$. Then $M$ has a natural structure as a (right) $R$-module via

$$
(a+B)(l+A)=[a, l]+B
$$

for all $a \in A$ and $l \in L$.

The $R$-module structure on $M$ carries over to an $R \otimes R$-module structure on the tensor square $M \otimes M$. There is an algebra homomorphism $\delta: R \rightarrow R \otimes R$ given by $x \delta=x \otimes 1+1 \otimes x$ for all $x \in L / A$. In fact, as is well known, $\delta$ is an embedding (it has right inverse $\iota \otimes \varepsilon$ where $\iota: R \rightarrow R$ is the identity map and $\varepsilon: R \rightarrow K$ is the augmentation map). We call $\delta$ the diagonal embedding. Let $\widetilde{R}=R \delta$. Thus $M \otimes M$ is an $\tilde{R}$-module, and therefore an $R$-module. The action of $R$ on $M \otimes M$ is called the diagonal action. It induces an action of $R$ on the exterior square $M \wedge M$ given by

$$
(m \wedge n) x=(m x) \wedge n+m \wedge(n x)
$$

for all $m, n \in M$ and $x \in L / A$. The action of $L$ on itself carries over to an action of $R$ on $B /[B, A]$ via

$$
(b+[B, A])(l+A)=[b, l]+[B, A]
$$

for ali $b \in B$ and $l \in L$. There is a linear map $\gamma: M \wedge M \rightarrow B /[B, A]$ satisfying

$$
\left(a_{1}+B\right) \wedge\left(a_{2}+B\right) \mapsto\left[a_{1}, a_{2}\right]+[B, A]
$$

for all $a_{1}, a_{2} \in A$, and it is easily verified (via the Jacobi identity) that $\gamma$ is a homomorphism of $R$-modules.

LEMMA. With the notation above, suppose that $L$ is finitely presented and that $L / A$ is of finite dimension. Then the kernel of $\gamma$ is a finitely generated $R$-module.

Proof: It is possible to prove this by means of the spectral sequence associated to the extension $A \rightarrow L \rightarrow L / A$, but we provide an elementary proof. Let $F$ be a finitely 
generated free Lie algebra such that there is an epimorphism $\pi: F \rightarrow L$. Let $U, V$ and $W$ denote the complete inverse images under $\pi$ of $A, B$ and $\{0\}$, respectively. Note that we can then identify $L / A$ with $F / U$ and hence $R$ with $K[F / U]$.

The correspondence given by

$$
\left(u_{1} \pi+B\right) \wedge\left(u_{2} \pi+B\right) \mapsto\left[u_{1}, u_{2}\right]+[V, U],
$$

for all $u_{1}, u_{2} \in U$, leads to an explicit $R$-module isomorphism between $M \wedge M$ and $U^{\prime} /[V, U]$. For details, see [6, Section 2.2]. The epimorphism $\pi$ also induces an isomorphism of $B /[B, A]$ with $V /([V, U]+W)$, and this is again an $R$-module isomorphism. We can thus identify $\gamma$ with the map

$$
U^{\prime} /[V, U] \rightarrow V /([V, U]+W)
$$

induced from the inclusion of $U^{\prime}$ into $V$. Therefore

$$
\begin{aligned}
\operatorname{ker} \gamma & \cong\left(U^{\prime} \cap([V, U]+W)\right) /[V, U]=\left([V, U]+\left(U^{\prime} \cap W\right)\right) /[V, U] \\
& \cong\left(U^{\prime} \cap W\right) /\left([V, U] \cap\left(U^{\prime} \cap W\right)\right)=\left(U^{\prime} \cap W\right) /([V, U] \cap W) .
\end{aligned}
$$

We must show that this section of $F$ is finitely generated as an $R$-module.

Since $L$ is finitely presented, $W$ is finitely generated as an ideal of $F$. Hence $W /[W, U]$ is finitely generated as an $R$-module. But $\left(U^{\prime} \cap W\right) /([V, U] \cap W)$ is isomorphic to an $R$-section of $W /[W, U]$. Since $L / A$ is of finite dimension, $R$ is Noetherian (see, for example [5, Proposition 6 of I.2.6]) and so this section is also finitely generated, as required.

Observe that this lemma, although technical in nature, has some important consequences in special cases. For example, if $B /[B, A]$ is finite dimensional, then we may deduce that $M \wedge M$ is finitely generated as an $R$-module and so, using [6, Theorem A], that $L / B$ is also finitely presented. The following is another special case where we can deduce that $L / B$ is finitely presented.

Proposition. Let $L$ be a finitely presented centre-by-metabelian Lie algebra over the field $K$. Let $A$ and $B$ be ideals of $L$ with $B \subseteq A$ such that $L / A$ and $A / B$ are Abelian and $B$ is central; and write $M=A / B$. Then $M \wedge M$ is finitely generated as a $K[L / A]$-module. As a consequence, $L / B$ is finitely presented (so, taking $B=L^{\prime \prime}$, we have that $L / L^{\prime \prime}$ is finitely presented).

Proof: Observe that the last sentence of the Proposition follows from [6, Theorem A]. Write $R=K[L / A]$ and let $I$ denote the augmentation ideal of $R$ (that is, the ideal of $R$ generated by the elements of $L / A$ ). By the Lemma, the kernel of $\gamma: M \wedge M \rightarrow B$ 
is finitely generated as an $R$-module. Since $B$ is central in $L$ it is trivial as an $R$ module (by which we mean that each element of $L / A$ has zero action on $B$ ). Thus the $R$-module $(M \wedge M) I$ is contained in the kernel of $\gamma$. Therefore, by the Lemma, it is finitely generated. We shall use this to show that $M \wedge M$ is finitely generated as an $R$-module.

By [6, Lemmas 2.1 and 2.2], we may assume that $K$ is algebraically closed. We shall use arguments similar to those of [6, Proposition 2.4]. By [4, Theorem 1 of IV.1.4], $M$ has a finite series of submodules

$$
\{0\}=M_{0} \leqslant M_{1} \leqslant M_{2} \leqslant \ldots \leqslant M_{k}=M,
$$

where each quotient $M_{i} / M_{i-1}$ is isomorphic to an $R$-module of the form $R / P_{i}$ where $P_{i}$ is a prime ideal of $R$. Further, by [4, Theorem 2 of IV.1.4], each $P_{i}$ contains a prime ideal $Q_{i}$ of $R$ which is associated to $M$.

It will clearly suffice to show that $M \otimes M$ is finitely generated as an $R$-module under the diagonal action. But the series above for $M$ yields a finite series of $R$ submodules of $M \otimes M$ in which each quotient is of the form $R / P_{i} \otimes R / P_{j}$ (here, of course, $R$ acts via the diagonal embedding of $R$ into $R \otimes R$ ). Since $R / P_{i} \otimes R / P_{j}$ is a quotient of $R / Q_{i} \otimes R / Q_{j}$, it suffices to prove that each $R / Q_{i} \otimes R / Q_{j}$ is finitely generated as an $R$-module.

Suppose firstly that $R / Q_{i} \otimes R / Q_{j}$ is trivial as an $R$-module. Then, for each $x \in L / A$,

$$
0=\left(\left(1+Q_{i}\right) \otimes\left(1+Q_{j}\right)\right) x=\left(x+Q_{i}\right) \otimes\left(1+Q_{j}\right)+\left(1+Q_{i}\right) \otimes\left(x+Q_{j}\right) .
$$

But this implies that $x+Q_{i} \in K+Q_{i}$ and $x+Q_{j} \in K+Q_{j}$ for each $x \in L / A$, so that $R / Q_{i}$ and $R / Q_{j}$ have dimension 1 . It is then clear that $R / Q_{i} \otimes R / Q_{j}$ is finitely generated as an $R$-module.

Thus we can assume that $R / Q_{i} \otimes R / Q_{j}$ is not trivial as an $R$-module. Choose an element $x$ of $L / A$ which has non-zero action on $R / Q_{i} \otimes R / Q_{j}$. We observe for future reference that, because $K$ is assumed algebraically closed and because $R / Q_{i}$ and $R / Q_{j}$ are integral domains, $R / Q_{i} \otimes R / Q_{j}$ is also an integral domain (see [9, Corollary 1 to Theorem 40 of Chapter III]). Thus multiplication in $R / Q_{i} \otimes R / Q_{j}$ by the image of $x$ is a monomorphism of $R$-modules.

Suppose that $Q_{i} \neq Q_{j}$. Because $Q_{i}$ and $Q_{j}$ are associated prime ideals of $M$, there are elements $m_{i}$ and $m_{j}$ of $M$ such that the submodules $m_{i} R$ and $m_{j} R$ are isomorphic to $R / Q_{i}$ and $R / Q_{j}$, respectively. Further, because $Q_{i}$ and $Q_{j}$ are distinct, these submodules intersect trivially, and so $m_{i} R+m_{j} R \cong R / Q_{i} \oplus R / Q_{j}$. Since $R / Q_{i} \otimes R / Q_{j}$ is isomorphic to a submodule of $\wedge^{2}\left(R / Q_{i} \oplus R / Q_{j}\right)$, it follows that $R / Q_{i} \otimes R / Q_{j}$ is 
isomorphic to a submodule of $M \wedge M$. Therefore $\left(R / Q_{i} \otimes R / Q_{j}\right) x$ is isomorphic to a submodule of $(M \wedge M) I$ and is finitely generated. But

$$
R / Q_{i} \otimes R / Q_{j} \cong\left(R / Q_{i} \otimes R / Q_{j}\right) x .
$$

Thus $R / Q_{i} \otimes R / Q_{j}$ is finitely generated.

Suppose now that $Q_{i}=Q_{j}$. Because $Q_{i}$ is an associated prime ideal of $M$, there is an isomorphic copy of $R / Q_{i}$ in $M$. Thus $\left(R / Q_{i} \wedge R / Q_{i}\right) x$ is isomorphic to a submodule of $(M \wedge M) I$ and is finitely generated. It is standard, and easily verified, that the linear map induced by $a \wedge b \mapsto a \otimes b-b \otimes a$ (for all $a, b \in R / Q_{i}$ ) yields an $R$-monomorphism from $R / Q_{i} \wedge R / Q_{i}$ to $R / Q_{i} \otimes R / Q_{i}$. Thus multiplication in $R / Q_{i} \wedge R / Q_{i}$ by the image of $x$ is a monomorphism of $R$-modules. Therefore $R / Q_{i} \wedge R / Q_{i}$ is isomorphic to $\left(R / Q_{i} \wedge R / Q_{i}\right) x$ and is finitely generated. It follows, by [6, Theorem $\left.\mathrm{A}\right]$, that $R / Q_{i} \otimes R / Q_{i}$ is finitely generated as an $R$-module, which completes the proof of the Proposition.

\section{Proof of The TheOREM}

We use the notation preceding the statement of the Lemma with $A=L^{\prime}$ and $B=L^{\prime \prime}$. Here $L^{\prime \prime}$ is central in $L$. It will sometimes be convenient to consider $M \wedge M$ and $M \otimes M$ as $\widetilde{R}$-modules rather than $R$-modules (recall that $\widetilde{R}=R \delta \subseteq R \otimes R$ ). By the Proposition, $M \wedge M$ is finitely generated as an $\widetilde{R}$-module. Hence, by [6, Theorem A], $M \otimes M$ is also finitely generated as an $\tilde{R}$-module.

Let $\left\{w_{1}, \ldots, w_{k}\right\}$ be a finite generating set for $M \otimes M$ as an $R \otimes R$-module and, for $i=1, \ldots, k$, let $J_{i}$ be the annihilator of $w_{i}$ in $R \otimes R$. Further, let $J$ be the annihilator of $M \otimes M$. Thus $J=J_{1} \cap \cdots \cap J_{k}$ and

$$
(R \otimes R) / J_{i} \cong w_{i}(R \otimes R) \leqslant M \otimes M .
$$

Since $M \otimes M$ is finitely generated as an $\widetilde{R}$-module, so is $(R \otimes R) / J_{i}$. Thus $(R \otimes R) / J$ is also finitely generated as an $\tilde{R}$-module.

Let $\widetilde{I}$ be the augmentation ideal of $\widetilde{R}$ and let $\widehat{I}$ be the ideal of $R \otimes R$ generated by $\tilde{I}$. Then $(R \otimes R) /(\hat{I}+J)$ is both finitely generated and trivial as an $\widetilde{R}$-module and so is of finite dimension. Let $T=\{t \in R: t \otimes 1 \in \widehat{I}+J\}$. Then $T$ is an ideal of $R$ such that $R / T$ is of finite dimension.

Let $\sigma: M \otimes M \rightarrow L^{\prime \prime}$ be the homomorphism of $R$-modules satisfying

$$
\left(a_{1}+L^{\prime \prime}\right) \otimes\left(a_{2}+L^{\prime \prime}\right) \mapsto\left[a_{1}, a_{2}\right]
$$

for all $a_{1}, a_{2} \in L^{\prime}$. Since $L^{\prime \prime}$ is a trivial $R$-module, $(M \otimes M) \tilde{I}$ is contained in the kernel of $\sigma$. But

$$
M T \otimes M \subseteq(M \otimes M)(\widehat{I}+J)=(M \otimes M) \tilde{I}
$$


Thus $(M T \otimes M) \sigma=\{0\}$.

Let $H$ be the subspace of $L$ such that $L^{\prime \prime} \leqslant H \leqslant L^{\prime}$ and $H / L^{\prime \prime}=M T$. Since $T$ is an ideal of $R, H$ is an ideal of $L$. From the definition of $\sigma$ we find $(M T \otimes M) \sigma=$ $\left[H, L^{\prime}\right]$. Thus $\left[H, L^{\prime}\right]=\{0\}$ and, since $H \leqslant L^{\prime}$, it follows that $H$ is Abelian. Since $T$ is of finite co-dimension in $R$ and $M$ is a finitely generated $R$-module, $M T$ is of finite co-dimension in $M$. Thus $H$ is of finite co-dimension in $L^{\prime}$ and so also in $L$. Therefore $L$ is Abelian-by-finite-dimensional, which completes the proof of the Theorem.

\section{REFERENCES}

[1] R.K. Amayo and I. Stewart, Infinite-dimensional Lie algebras (Noordhoff, Leyden, The Netherlands, 1974).

[2] R. Bieri and R. Strebel, 'Almost finitely presented soluble groups', Comment. Math. Helv. 53 (1978), 258-278.

[3] R. Bieri and R. Strebel, 'Valuations and finitely presented metabelian groups', Proc. London Math. Soc. (3) 41 (1980), 439-464.

[4] N. Bourbaki, Commutative algebra (Addison-Wesley, Reading, U.S.A., 1972).

[5] N. Bourbaki, Lie groups and Lie algebras, (Part 1: Chapters 1-3) (Springer-Verlag, Berlin, 1989).

[6] R.M. Bryant and J.R.J. Groves, 'Finitely presented Lie algebras', J. Algebra (to appear).

[7] J.R.J. Groves, 'Finitely presented centre-by-metabelian groups', J. London Math. Soc. (2) 18 (1978), 65-69.

[8] A. Wasserman, 'A derivation HNN construction for Lie algebras', Israel J. Math. 106 (1998), 79-92.

[9] O. Zariski and P. Samuel, Commutative algebra, Volume I (Van Nostrand, Princeton, 1958).

Department of Mathematics

UMIST

Manchester M60 1QD

United Kingdom
Department of Mathernatics

University of Melbourne

Parkville Vic 3052

Australia 